\title{
ELK ASIA PACIFIC JOURNAL OF MECHANICAL ENGINEERING RESEARCH
}

Volume 1 Issue 1, September (2014)

\section{[] ELK Asia Pacific Journals}

www.elkjournals.com

\section{STUDY ABOUT CLOUD SCREENING ALGORITHMS FOR AEROSOL ROBOTIC NETWORK}

\section{Prof. Rajesh M}

Government College of Engineering

Amaravati, Maharashtra,

India

\author{
Dr. Gautam Kumar Jain \\ Mechanical Engineering Dept. \\ College of Engg \& Technology, \\ Kuwait
}

\begin{abstract}
Automatic globally distributed networks for supervising aerosol optical depth offer measurements of anthropogenic and natural aerosol loading which is essential in several regional and local studies as well as worldwide change research examinations. Such networks' strength is depends on imposing a standardization of processing and measurement permitting large scale and multiyear contrast. The growth of AERONET (Aerosol Robotic Network) for systematic sun photometer measurements based on ground of aerosol optical depth is a developing and important step in this process. The developing database needs the growth of a consistent, system wide and reproducible procedure of cloud screening. This study argues the justification and methodology of cloud screening algorithm evolved for database of AERONET. The procedure has been tested extensively on experimental data acquired in varied optical and geographical conditions. These conditions involve events of biomass burning in Zambia and Brazil, hazy conditions of summer in the area of Washington $D C$, clean air transport from Arctic of Canada and cloudy conditions variably. For different sites the screening algorithm of this study removes from -20 percent to 50 percent of initial information relying on conditions of cloud. Some shortcomings of the suggested procedure are argued.
\end{abstract}

Keywords: Cloud-screening, Quality control algorithms, AERONET

\section{Introduction:}

The spatial and temporal distribution of anthropogenic and natural loading of aerosol in atmosphere has acquired attention of sporadic scientific during the past $1 / 2$ of this century as a computation of regional air pollution and for uncertain influence on worldwide climate. During the past decade, larger understanding of aerosol processes and renewed interest has required an emphasis on supervising by satellite and ground based approaches of remote sensing.
Systems based on ground while regarded reliable, simple and essential to assist satellite retrieval methodology through programs of validation have lacked consistent long term assistance and endured from bad processing and measurement methodology resulting in fragmented sets of data that are difficult to utilize for scientific assessments (Forganet al, 1994). Numerous automatic measurement networks of aerosol have imposed a standardization of measurement (Forgan private 


\section{ELK Asia Pacific Journals}

www.elkjournals.com

communication, 1998; Holben et al, 1996; Takamura private communication, 1996; Michalsky et al, 1994, GAW, 1992) for their respective instruments. Processing, measurement and assurance of quality is developing for entire networks. One essential perspective of this evolutionary method similar to entire aerosol optical depth retrievals from networks of sun photometry is isolation of cloud influenced data from cloud free information. It is in principle much simple to handle with clouds presence for manual instruments. Human observers can predict clouds based on spatial patterns and subtle texture and therefore do not make examination under those situations (Kaufman and Fraser, 1963). Automatic instruments deployment poses the issue of referring an efficient procedure of cloud screening, in handling with more than one instrument for relatively small periods of time it is feasible to carry out subjective screening by human analyst (Markham et al, 1997; Smirnov et al, 1994; Abern et al, 1991). Further discussion for automatic cloud screening is that observers of human can be inconsistent in their decisions of cloud detection. Procedures must be computerized and similarly generalized as much as feasible to be capable to manage sets of data related with different kinds of aerosol. Harrison and Michalsky (1994) have evolved an algorithm of objective analysis for their regressions of Langley. This algorithm was configured for a multifilter rotating shadow band radiometer. Instruments of this kind have their own methodological individualities and hence need a varied protocol of measurement. The AERONET federated network is the most worldwide distributed system based on ground resulting in a database of vastly variable conditions of atmosphere. AERONET forces standardization for processing data, calibration and measurement protocol. A physically admissible and reliable automatic procedure of cloud screening is the basis for program's success. The automatic sky/sun CIMEL radiometer CE-318 needs information nevertheless of conditions of sky. The radiometer makes two general measurements either diffuse radiances of sky or direct sun radiances both within 


\section{ELK Asia Pacific Journals}

www.elkjournals.com

numerous programmed sequences. The direct measurements of sun pose the most critical screening issue and are the subject of this study. This study utilized two leading criteria in their procedure of cloud screening. First the author retained stable triplets to remove high frequency alterations. Second the author removed quick temporal optical depth diurnal differences between chosen triplets by using a second derivative threshold root mean square. The procedure was verified on experimental information acquired in varied optical and geographical conditions. On the whole with the exception of cases including thin stable cirrus the algorithm of cloud screening generated promising outcomes.

\section{Description of Algorithm:}

\section{Checks of data quality:}

If the optical depth of aerosol is reduced than -0.01 at any wavelength the author do not agree the corresponding $\tau_{\sigma}$. The author remove only measurements in that specific channel where $\tau_{\sigma}<-0.01$ while securing $\tau_{\sigma}$ in entire channels that yielded optical depths greater than -0.01 . The aerosol optical depth negative values are not physical. However these reduced optical depths might be affected by calibration, correction of temperature at 1.020 nanometer wavelength, column ozone and atmospheric pressure amount uncertainties. Measurements made during reduced elevation angles of sun have a greater opportunity of cloud contamination and so as not to weight daily averages unduly with greater frequency data obtained during the sequence of Langley (Holben et a, 1998) $\tau_{\alpha}(\boldsymbol{\lambda})$ for mass of air $m>5$ are not regarded in screened database.

\section{Criterion of Triplet Stability:}

A triplet measurement acquired with CIMEL sky/sun radiometer comprises of three measurements each made thirty seconds apart over a total of one minute period. The author presume that the aerosol optical depth in complete atmospheric column must differ by less than 0.02 within one triplet for entire wavelengths if the atmosphere is to be regarded cloud free and stable. When a triplet is recognized as better the author utilize the average $\tau_{\alpha}$ value of 3 


\section{ELK Asia Pacific Journals}

www.elkjournals.com

measurements as the cloud screened $\tau_{\sigma}$ value. If the variability of triplet exceeds threshold at any wavelength the author remove the calculation at entire wavelengths wholly. For the worst case scenario the highly variable aerosol optical situations may be regarded as a bad case scenario for the test of triplet. An individual cirrus cloud band was examined only once at around 1235 GMT. Due to greater loading of aerosol certain cirrus might have unpredicted but they would have been of similarly reduced optical depth. As one would anticipate for smoke aerosol the most variable channel seems to be 340 nanometer. At the largest wavelengths the optical depth of aerosol reveals much reduced variability. This is typical for biomass burning conditions since the distribution size is dominated by little particles. The author made extra tests of their criteria of triplet variability on measurements made in conditions of high aerosol loading and biomass burning. The most essential inference which the authors are capable to make is that their regarded threshold of 0.03 $\tau_{\sigma}$ permits numerous variability of triplet than needed in several cases.

\section{Check of diurnal stability:}

If the averaged optical depth of aerosol's standard deviation at 500 nanometer for a complete day is less than 0.015 then the author decline the screening and agree entire calculations. Because the measured accuracy of newly calibrated instruments of field is around +-0.01 in $\tau_{\sigma}$ it is not essential to verify the remaining data since the diurnal variability is reduced than or common to accuracy of measurement.

\section{Criteria of Smoothness:}

The criterion of smoothness is based on restricting the aerosol optical depth's root mean square $2^{\text {nd }}$ derivative with time. The first derivative provides the temporal change rate and the second derivative refers the tendency variability and consequently it is sensitive to optical depth local oscillations affected by clouds and the average second derivative develops considerably in existence of such oscillations. It must be indicated that restricting the physical 


\section{ELK Asia Pacific Journals}

www.elkjournals.com

features average derivatives utilized in constrained inversion methods in remote sensing. There such restrictions of smoothness are applied successfully in extracting size distribution of aerosol particle or vertical profiles of atmosphere to remove artificial local oscillations associated to effects of error. In this study's cloud screening algorithm the author have acquired the strategy of smoothness evaluation evolved in constrained inversion method.

\section{Discussion:}

Some shortcomings of the study's screening algorithm are evident. To make a scheme that is as usual as feasible the author did not provide any limitations on parameter of angstrom. A feasible check of contamination of cloud would be to confine the parameter of angstrom variability relying on site and prevailing kind of aerosol. One more check for data quality was performed specifically for greater turbidity conditions. In some cases when aerosol optical depth was generally greater than 1.5 an artifact of out of band leakage has been examined in smallest wavelengths. The parasitic light existence in signal of sun photometer led to afternoon and early morning drop offs in optical depth of aerosol's diurnal behavior. This simply refers the signal was greater than it must be and hence the evidently anomalous reduced optical depths of aerosol were recorded. The important difficulty induced by the parasitic light existence in a signal of sun photometer is that the optical depth over wavelength leakage band features can be varied essentially from optical depth related with nominal filter band. In handling with this issue on case by case fundamentals the author predicted that if the calculated voltage is reduced than fifty counts the measurements must be removed. After weak signals related with out of band leakage were eliminated, the diurnal variability is realistic and much less used to impact of this evident spectrally dependent artifact of spectrum. The scatter-gram reveals that the Angstrom parameter variability for cloud screened and unscreened instantaneous optical depth measurements of aerosol were taken in Bolivia Los Fierros from $4^{\text {th }}$ may till $1^{\text {st }}$ 


\section{ELK Asia Pacific Journals}

www.elkjournals.com

September 1996. This period of measurement encloses the burning and preburning seasons thus different optical conditions were examined. The author makes last comments considering their quality control and cloud screening algorithm. The evident contaminated data cloud was removed since cloud denotes parameter values of Angstrom near 0 or even negative due to big crystal/droplet sizes. The author prefer to emphasize that they do not yield particular restrictions on parameter of Angstrom but only on optical depth's temporal variability. Approximately 75 percent of beginning data remained after the screening of cloud. This study handles with the cloud screening and quality control components for a particular instrument and for particular database and related sequence of measurement. Therefore this is not a generalized process which would be used to entire kinds of $\tau_{\sigma}$ systems of measurement. Schmidt et al (1999) reveal that contract in optical depth of aerosol calculated by CIML and other instruments in conditions of field experiments is within $0.015 \mathrm{rms}$. Holben et al (1998) and Eck et al (1999) provide overall accuracy's careful assessments of CIMEL sun-photometer due to uncertainty of calibration and lack of pressure data surface and actual amount of ozone column. The last accuracy relies in part on field history involving optical, mechanical and electrical integrity during the field calculations.

\section{Summary:}

The major inferences drawn from this research can be summarized below:

1. An algorithm of cloud screening for optical depth aerosol database of AERONET was made, implemented and tested comprehensively. The two major criteria of threshold are both associated to temporal differences of $\tau_{\sigma .}$. One is used to small variability time period and the other is used to diurnal and hourly time period differences of $\tau_{\sigma}$.

2. The conditions imposed on optical depth diurnal variability of aerosol are not huge number and do not bias the daily averages computation 


\section{ELK Asia Pacific Journals}

$\underline{\text { www.elkjournals.com }}$

strongly. The suggested algorithm can be used to any kind of AERONET network in as most of the paradigm was evolved across an ensemble of aerosol conditions and sites of network.

3. Since temporal differences of $\tau_{\sigma}$ are recognized as contamination of cloud it is stated that certain variable aerosol plumes cases will be screened by this algorithm. Conversely stable equal cloud will surpass the thresholds of algorithm and be recognized as cloud free. However the author emphasize that the actual AERONET database sites is accessible in internet.

\section{References:}

[1] Eck, T. F., Holben, B. N., Reid, J. S., Dubovik, O., Smirnov, J. A., O’Neill, N. T., Slutsker, I., and Kinne, S. (1999), The wavelength dependence of the optical depth of biomass burning, urban and desert dust aerosols. J. Geoph. Res. Southern California perspective of the April, 1998 trans- 104:31,333-31,350.

[2] Forgan, B. W., DeLuisi, J. J., Hicks, B. B., and Rusina, E. N. (1994), Report on the measurements of atmospheric tur- pp. 137-140. bidity in BAPMoN, Rep. WMO, No. 94, Geneva.

[3] Holben, B. N., Eck, T. F., Slutsker, I., Tanre, D., Buis, J. P., a. One (the Setzer, A., Vermote, E., Reagan, J. A., Kaufman, Y. J., Nakajima, T., Lavenu, F., Jankowiak, I., and Smirnov, A. period variability (1 minute) and the other (1998), AERONET - A federated instrument network and (smoothness criterion) to hourly and diurnal time data archive for aerosol characterization. Rem. Sens. Env. period variations of sa. 66:1-16.

[4] Kaufman, Y. J., and Fraser, R. S. (1983), Light extinction by diurnal variability are not excessive and do not aerosols during summer air pollution. J. Clim. Appl. Met. 22:1694-1706.

[5] Markham, B. L., Schafer, J. S., Holben, B. N., and Halthore, R. N. (1997), Atmospheric aerosol and water vapor characteristics over north central Canada during BOREAS. J.network sites and aerosol conditions. Geoph. Res. 102:29,737-29,745.

[6] Measurement of the seasonal and annual variability of total column aerosol in a northeastern US network. In Proceed NET

[7] Michalsky, J. J., Schlemmer, J. A., Larson, N. R., Harrison, L. C., Berkheiser III, W. E., and Laulainen, N. S. (1994), 


\section{ELK Asia Pacific Journals}

www.elkjournals.com

[8] Schmid, B., Michalsky, J., Halthore, R., Beauharnois, M., Haraerosols L., Livingston, J., Russell, P. B., Holben, B. N., Eck,

[9] sites Vol. A, Snowbird, UT, September 26-30, Pittsburgh, PA, pp. 247-258.

[10] Smirnov, A., Royer, A., O’Neill, N. T., and Tarussov, A. (1994), A study of the link between synoptic air mass type and atmospheric optical parameters. $J$. Geophys. Res. 99:20,967-

[11] T. F., and Smirnov, A. (1999), Comparison of aerosol optical surements. Appl. Opt. 30:5276-5287. 